Sabina Leonelli, ESRC Centre for Genomics in Society, Department of Sociology and Philosophy, University of Exeter, s.leonelli@exeter.ac.uk

Published in Biological Theory, 2012

\title{
Classificatory Theory in Biology
}

Abstract: Scientific classification has long been recognized as involving a specific style of reasoning and doing research, and as occasionally affecting the development of scientific theories. However, the role played by classificatory activities in generating theories has not been closely investigated within the philosophy of science. I argue that classificatory systems can themselves become a form of theory, which I call classificatory theory, when they come to formalize and express the scientific significance of the elements being classified. This is particularly evident in some of the classification practices used in contemporary experimental biology, such as bio-ontologies used to classify genomic data and typologies used to classify 'normal' stages of development in developmental biology. In this paper, I explore some characteristics of classificatory theories and ways in which they differ from other types of scientific theories and other components of scientific epistemology, such as models and background assumptions.

Keywords: theory; biology; classification; data; bio-ontologies. 


\section{Introduction: Classification as theory-making}

The epistemic role of classificatory practices, long regarded as a conceptually uninteresting part of scientific research, is currently being reconsidered. Albert Crombie (1994) and Ian Hacking (2002) have argued that scientific classification involves a specific style of reasoning which is central to scientific research. John Pickstone has also recognized natural history, and the classificatory practices therein, as a unique "way of knowing": in his words, "knowing the variety of the world" by "collecting, describing and displaying" (Pickstone 2000: 60). Pickstone rightly emphasizes the broad historical and cultural context of classification in natural history, which include a variety of motives such as "pride of possession, intellectual satisfaction and commerce and industry" (ibid: 60); similar themes have been extensively discussed in sociological approaches to biology (e.g. Bowker and Star 1999). Here I wish instead to focus on the epistemic results of classification. Staffan Müller-Wille (2007, 2012 with Charmantier) and Bruno Strasser (2011, and 2011 with de Chadarevian) have provided extensive historical studies of the ways in which classificatory work ranging from $18^{\text {th }}$ century taxonomy to $20^{\text {th }}$ century molecular biology has involved both experimental and descriptive activities, collection as well as explanation, observation as well as analysis. Müller-Wille in particular has pointed to the role of classificatory practice in shaping ontological commitments and providing conceptual scaffolding to biological research. In a similar vein, Ursula Klein has examined the ways in which the diversity of classification systems populating $18^{\text {th }}$ century chemistry was used by historical actors to highlight wide differences in ontological beliefs (Klein and Lefèvre 2007), and Lorraine Daston has written about taxonomic practice as 'metaphysics in action' (Daston 2004: 158). On the philosophy front, John Dupré has articulated the growing consensus around the peculiar 
epistemic role of classification by pointing out that "classification in biology has a life of its own" (Dupré 2001), and that classification systems such as taxonomies and phylogenies profoundly affect the ways in which organisms are conceptualized in biology (Dupré and O’Malley 2007). Here I intend to go further than these claims, by arguing that classificatory practices can sometimes become deeply theoretical activities -- not merely theory-laden, but theory-making; and that this way of theorizing is an important component of biological reasoning, to be distinguished from other elements of theory such as law-like generalizations (like Hardy-Weinberg's law of population equilibrium), explanatory principles (such as selection in evolutionary theory), mechanisms (e.g. descriptions of how DNA replication takes place), or modeling activities (for instance, the use of graphs to illustrate metabolic networks - although admittedly, these would count as forms of theorizing only under a semantic account of theories). In this paper, I sketch some key characteristics of classificatory theories and focus on their role in experimental research - what it is that they do and do not enable scientists to do.

For the purposes of my argument, I shall use a conception of theory-making as a fundamental practice in science, which is enmeshed and intertwined with other practices such as data collection, experimental intervention and modeling, but which can be analytically distinguished from them. The vision of scientific research that most closely resonates with this view is James Griesemer's "house of science", and particularly his view of scientific inquiry as a set of activities of exploration whose purpose is to understand natural phenomena through the production of data, models and theories (Griesemer 2000). I am particularly interested in Griesemer's framing of data production and collection as tracking activities, whose material result is the generation of objects that can be treated as documents of the phenomena being 
tracked (Griesemer 2006). This is a material conception of data consonant with Hans-Jörg Rheinberger's and Ian Hacking's idea of data as "traces" and "marks" (Hacking 1992, Rheinberger 2011), which I analyzed in some detail elsewhere (Leonelli 2009). ${ }^{1}$ It is also a view that emphasizes the importance of scientists' commitments, both practical (to instruments and materials) and theoretical (to specific conceptualizations and background knowledge), in conducting tracking activities and interpreting their products.

What I want to consider here is the ways in which tracking activities generate theoretical commitments that sometimes become so formalized and influential as to deserve the status of 'theory'. My broad motivation is to explore the relation between tracking and theorizing activities in science, and the ways in which these activities inform each other. I view classificatory practices within experimental biology as an ideal locus for an analysis of the relation between scientific tracking and theorizing. All acts of classification, whether in or out of science, involve the grouping and labeling of objects in ways that are theoretically motivated as well as empirically informed by the tracking methods used to collect them. Within experimental biology, two characteristics lend classificatory activities additional epistemic power. On the one hand, classification aims at providing a representation of natural phenomena, and thus needs to conform to requirements for internal consistency, (relative) stability and empirical accuracy; on

\footnotetext{
${ }^{1}$ In my interpretation of this view, any material product of research activities, ranging from artefacts such as photographs to symbols (e,g, numbers), can be considered as a piece of data as long as (1) it is taken to represent an aspect of the phenomenon under investigation; and (2) it is possible to disseminate it across a community of scientists (the manipulation of artefacts aimed at their dissemination is also identified by Rheinberger, with a nod to Bruno Latour's views on immutable mobiles, as crucial to transforming 'traces' into 'data'; Rheinberger 2011, 344). Further, note that I am here treating all data, no matter whether in a digital or in a material form, as material artifacts. The reason for this is that digital artefacts are concrete objects, even if the physical constrains and the type of resistance offered by virtual environments are different from those encountered in non-virtual situations (data in a digital format are only visible and manipulable via interaction with computer screens). This position, which I expand upon in forthcoming work, is compatible with Wendy Parker's idea of 'computer experiments as, first and foremost, experiments on real material systems' (2009: 488).
} 
the other hand, the criteria chosen for classification need to take account of its practical use in an experimental context, and particularly of how the suggested grouping of objects may inform and facilitate experimental intervention. The objects to be classified in this context comprise both phenomena of interest to scientists and the data produced to track them. Indeed, my analysis will build on the ambiguous relationship between data and phenomena, and on the importance of classificatory categories in interpreting data as evidence for claims about phenomena.

To give flesh to these ideas, I shall first briefly describe two classification systems used to handle genomic data in model organism databases and morphological data in developmental biology. I will then argue that these classification systems should be recognized as a specific type of theory, classificatory theory, which differs from other forms of theory more typically encountered in the philosophy of science literature. In closing, I shall discuss the stakes of my argument, and particularly why it is important to consider some classificatory outcomes as theories rather than as other components of scientific inquiry (such as models, instruments or background knowledge).

\section{Classificatory activities in contemporary biology}

In this section, I briefly review two cases of classificatory activities in biology which exemplify my general argument on the theory-making power of classification. The first is the case of bioontologies, classification systems primarily used to store, organize and retrieve data acquired from biological materials via digital databases accessible through the internet. The Gene 
Ontology, one of the best developed bio-ontologies to date, was developed for use within community databases as a standard for the annotation of gene products deriving from research on model organisms (Ashburner 2000, Leonelli 2010, 2012a). A chief characteristic of bioontologies such as the Gene Ontology is their use of biologists' own research interests as a basis for classification. Bio-ontologies classify data through terms that refer to the phenomena for which those data are supposed to provide evidence (where by phenomena I mean the highly structured, experimentally situated labels given by researchers to their research objects, as described in Bogen and Woodward $1988^{2}$ ). In other words, the choice of classificatory categories is based on the existing research interests of experimenters and on available conditions for collecting and analyzing the data in question. At the same time, given the increasing need for wide dissemination of genomic data across research contexts, bio-ontologies are constructed to work across different model organism communities and separate disciplines. This means that the terms that they employ for classification have to be intelligible to as many biologists as possible, regardless of their specialist training or epistemic culture. To serve this purpose, bio-ontology terms, and their relation to each other, are defined very clearly and precisely, thus coming to constitute "formal representations of areas of knowledge" (Bard and Rhee 2004). For instance, the term "double-strand break repair via homologous recombination" is defined as "the error-free repair of a double-strand break in DNA in which the broken DNA molecule is repaired using homologous sequences. A strand in the broken DNA searches for a homologous region in an intact chromosome to serve as the template for DNA synthesis. The restoration of two intact

\footnotetext{
${ }^{2}$ Bogen and Woodward were famously ambiguous about their definition of phenomena, which can be taken to denote features of the world as well as the labels given to those features by researchers (e.g. McAllister 2007). For the purposes of my discussion, I am happy to maintain this ambiguity so that bio-ontologies can be interpreted as capturing real objects or the ways in which biologists describe those objects. The realism of bio-ontologies is hotly debated in applied ontology circles (e.g. Smiths and Ceusters 2010), and taking a position on this discussion is not relevant to my purposes here.
} 
DNA molecules results in the exchange, reciprocal or nonreciprocal, of genetic material between the intact DNA molecule and the broken DNA molecule" (Gene Ontology Website, accessed December 2011).

Bio-ontology terms thus act both as standards and as regulatory ideas: they enable communication of and about genomic datasets by homogenizing a plurality of activities and meanings under one conceptual umbrella, which makes it possible to develop future research with a shared basis. When depicted in this way, bio-ontologies seem to behave like any other classification system, providing labels that conceptually structure and map a field of inquiry. One further characteristic of bio-ontologies, however, adds a spin to their epistemic status: the biological knowledge expressed through bio-ontologies is best understood when assessed with reference to the specific datasets being classified, as well as the means and materials used to acquire those data. Let me briefly elaborate on this important point. Bio-ontology terms are selected and defined with the purpose of facilitating the circulation and retrieval of existing bodies of evidence. In so doing, they provide a representation of the knowledge about biology that is usually assumed as background when circulating data: further, they formalize that knowledge so that it can be explicitly formulated as a set of claims about biological entities and processes, and used to assess and interpret the significance of specific datasets. Such a representation is not meant to be a complete, self-standing representation of what is known about those entities and processes. Rather, it is intended to capture the knowledge needed to use data already available on those entities and processes; and its significance is best interpreted in relation to the methods, materials and instruments used to track those entities and processes. This is why bio-ontology curators strive to include information about the provenance of data, usually 
referred to as "meta-data", in the databases that use bio-ontologies as classification systems. For instance, the Gene Ontology uses the term "double-strand break repair via homologous recombination" to capture data about Breast cancer type 1 susceptibility protein (BRCA1), and includes information about the type of experiment through which the data were generated, the paper where this was first described, and the group that made the annotation (in this case, the HUGO Gene Nomenclature Committee).

The second case I wish to consider is what Alan Love describes as "typological thinking" in developmental biology and systematics, which in his words involves "representing and categorizing natural phenomena, including both grouping and distinguishing these phenomena according to different characteristics, as well as ignoring particular kinds of variation" (Love 2009: 53). A good instance of this is the choice and use of specific descriptions and/or representations of normal stages of development as classification tools for data in developmental biology. Loves makes a strong argument, partly built in relation to other recent work on the importance of natural kinds as classification tools (Dupré 1993 and 2001, Brigandt 2009, Reydon 2010), that the typologies used to classify stages of development are chosen partly on pragmatic grounds, such as familiarity with the types and formats of relevant data and the strategies through which those data are collected; and partly on the basis of expectations about which aspects of the organisms in question will be of most relevance to advancing existing scientific knowledge about developmental processes. For instance, developmental biologists select images or descriptions exemplifying stages in the post-embryonic development of chick or frog foetuses on the basis of their experience of what constitutes a "typical stage" across multitudes of different specimens (which in turn involves familiarity with the ways in which specimens are studied and the specific 
kinds of data acquired through those processes) and of their background expectations about how images of foetuses at different stages are collected and interpreted in their field (Minelli 2003). These are choices about tracking, i.e. about how to acquire material documents (data) enabling the investigation of biological processes of interest (in this case, the development of a given organism). These choices notoriously have descriptive as well as normative undertones: "[the classification of] normal stages involve assessments of 'typicality' because of enormous variation in the absolute chronology of different developmental processes" (Love 2009: 63; see also Griesemer 1996 for similar arguments). They embody specific assumptions about how to track and provide stable representations of an essentially dynamic and hugely species-specific process (e.g. what constitutes adequate data in the research programs in question), which result in knowledge about the very processes being investigated (e.g. statements identifying causal connections between developmental stages and homologies between different species) which is then used to evaluate and interpret available data.

Classificatory categories such as developmental stages embody and formalize these choices and assumptions, and thus come to express substantive decisions about how development will be measured; which parameters count, and why; and which terminology and methods best encapsulate available knowledge on organismal development, while at the same time facilitating future efforts of data collection and interpretation. So, on the one hand, these classificatory systems work at least partly because of their efficiency as tools to co-ordinate the collection, dissemination and analysis of data on development; on the other hand, they constitute themselves conceptualizations of development which have consequences for how research in this area is carried out, and on what. In order to adequately assess the import and significance of such 
conceptualizations, biologists typically evaluate the experimental context in which they were originally proposed. This provides a way to understand the pragmatic motivations for specific conceptualizations, and to assess their usefulness when applied in other experimental contexts.

\section{The features of classificatory theories}

Classification practices such as the ones depicted above co-ordinate and underlie the tracking of biological phenomena and the collection of the resulting data into representations that can be used to model those phenomena. In doing so, they offer a substantive formalization of biological knowledge about organisms, whose primary goal is to facilitate the interpretation and further collection of data about organismal structures and development across species. These formalizations uncover knowledge that is given for granted when collecting, disseminating and using data. Further, by assembling, integrating and expressing knowledge used to handle data, these classification systems make a new and unique contribution to scientific research: they express knowledge that cannot be found anywhere else (models, instruments, existing theories or textbooks). This is not necessarily the same as contributing entirely new knowledge, in the sense of capturing new discoveries: for instance, bio-ontologies tend to integrate and formalize knowledge that already exists, but is dispersed in different areas of biological research (such as different model organism communities, or different subdisciplines working on the same phenomenon). This formalization requires extensive conceptual work, and often constitutes an important step forward in the development of biological knowledge, even if it does not necessarily incorporate paradigm-shifting discoveries. For instance, the Gene Ontology has 
developed a new definition of the term 'gametogenesis' that takes account of the differences between this process in plants and animals, which researchers were able to articulate clearly thanks to this classificatory practice (for more details and examples, see Leonelli et al 2011).

This example shows how classification systems such as the Gene Ontology are not the same as a simple list of items, where existing materials are ordered according to an explicit, pre-existent conceptual criterion (as in the case of biodiversity surveys for instance, where an inventory of biodiversity is constructed around a specific species criterion; or botany manuals, where observers of plants are invited to identify them according to pre-existing categories). The process of ordering alone does not amount to theorizing, and often in science things are ordered according to pre-established criteria, a process that does not generate significant conceptual implication. When ordering is intertwined with the analysis and interpretation of the scientific meaning of data, something more is at stake. The criteria for what count as good terms, definitions and links with data within the Gene Ontology, and thus for what biological knowledge is expressed in the system, are developed as part and parcel of the process of classification. As a result of this process, classification systems such as bio-ontologies and developmental stages are more than just theory-laden: they express criteria to evaluate and interpret the scientific significance of the items which they are used to classify. This is knowledge that underpins and directs scientific practice, the production of new data and, most importantly, debates about the structure and functioning of biological entities and processes. In this sense, classificatory systems such as bio-ontologies and stages of development constitute a form of theory, which I shall henceforth refer to as classificatory theory. Viewing these systems as theories recognizes their crucial epistemic role in expressing the biological knowledge 
underlying experimental research, while at the same time emphasizing the fallible, dynamic and context-dependent nature of such knowledge. ${ }^{3}$ Like Quine's webs of beliefs, classificatory theories face the "tribunal of experience": they are regularly challenged by new evidence and can be modified and updated when necessary. Recognizing the role of bio-ontologies as theories uncovers and highlights the conceptual substance and commitments underlying their adoption, thus alerting biologists against an uncritical use of databases which use these classification systems. Similarly, the identification of types used in developmental biology as forms of theory enables their critical discussion and, where necessary, questioning in the face of contradictory conceptualizations or experimental results. As stated by three prominent developmental biologists discussing the status of developmental stage as tools for data analysis in vertebrates: "thinking in terms of types, either as developmental stages or as putative ancestors, can be helpful in searching for order in the diversity of animal life. However we need to be aware of the limitations of typologism" (Richardson et al. 1998).

In short, I am not arguing here that all classification systems need to be viewed as sources for classificatory theories. What I am arguing is that classifications can play the role of theories depending on the extent to which they embody and express a specific way of interpreting the overall significance of a set of empirical results.

\section{How classificatory theories differ from other forms of theory}

\footnotetext{
${ }^{3}$ The dynamism of these types of theories might be best captured with reference to John Dewey's account of the process of inquiry, in which knowledge is continually constituted and recreated through the process of scientific investigation, and the very attempt to formalize knowledge into theories works as a map and as an enabling condition for such change (Dewey 1938).
} 
I now turn to the status of classificatory theories vis-à-vis other forms of scientific theorizing, such as for instance law-like generalizations. I confront this issue by discussing how some of the main characteristics that are typically attributed to scientific theories apply to the case of classificatory theories. As I show, each of these characteristics fits the case of classificatory theories, and yet it is realized in ways that differ substantially from other notions of theory.

\section{(1) Generalizing}

Classificatory theories aim towards generality in the sense that they provide common labels covering a number of phenomena and related research results. These general labels are used to interpret the evidential value of new datasets, provide heuristic guidance and conceptual structure to future investigations and contribute to biologists' understanding of phenomena. Their level of generality is, however, not fixed, and far from aspirations to universality:

- It is not fixed because the scope of application of classificatory theories can vary greatly depending on the research context and objects on which they are used, as well as the scientist(s) using them (thus mirroring the notion of formalism proposed by Griesemer in this special issue). On the one hand, the significance and epistemic value of classificatory theories is domain-dependent: they can be accepted or challenged depending on the research context in which they are used. On the other hand, the meaning and intelligibility of these theories depends on their user's knowledge of the scientific practices through which they were developed: that is, knowledge of the materials, settings and techniques through which their objects - the phenomena which they posit and characterize - have been tracked. In other words, the meaning and 
epistemic value assigned to theoretical claims made as a result of classificatory practices depends on one's expertise in tracking the phenomena in question. ${ }^{4}$

- And it is far from aspiration to universality, because classificatory theories tend to accrue generality over narrowly defined domains. For instance, both the Gene Ontology and stages of development aim to generalize over species, by making classificatory categories applicable beyond the species on which data were originally obtained. This aspiration manifests itself differently depending on which species are targeted. Different classifications of stages of development tend to cover species that are phylogenetically close, such as specific families of vertebrates. Within the Gene Ontology, generalizing over species has meant generalizing over the most popular model organisms on which molecular data are being gathered (so the principal aim is generalization over Arabidopsis thaliana, C. elegans, Drosophila melanogaster and Mus musculus - no small endeavour of course, given the diversity among these organisms ${ }^{5}$ ).

\section{(2) Unifying}

Margaret Morrison's 2007 account of theoretical unification, which the diversity of scientific practices into account, proves very useful in clarifying the kind of unity that classificatory theories aim to foster. This is what Morrison calls reductive unity (as opposed to synthetic unity):

\footnotetext{
${ }^{4}$ Krakenau et al note that 'One of the vaunted benefits of machine learning is that classification and prediction tasks can be performed without insights into the structure and dynamics of the underlying system' (2011: 272). When considering bio-ontologies, one of the main motors of machine learning, as a form of theory, it is clear that this conceptualisation of machine learning does not hold. Classification is a highly conceptual exercise, whose value and significance can only be assessed through knowledge of the underlying biological system.

${ }^{5}$ One could question the extent to which this generalisation has been successfully achieved. While I do not see this as crucial to my argument, which concerns the underlying aspiration of this system to generalise rather than its success in doing so, I have discussed the successes and difficulties of this enterprise in Leonelli et al 2011 and Leonelli 2012 b.
} 
an ideal of unity which only aims to establish some kind of commonality between different phenomena, without necessarily embedding that commonality within an overarching conceptual structure. Indeed, systems such as bio-ontologies aim to achieve classificatory categories that highlight similarities between different species, and accordingly bio-ontology curators devote much of their time to standardising terminology in ways that fit different biological subcultures. This reductive unification is not coupled with the attempt to achieve synthetic unity. Rather, classificatory systems such as bio-ontologies or developmental stages aim to enable scientists to pursue disunified, fragmented research about a large variety of objects. Classification systems tend to achieve reductive unity by abstracting from specific instances of entities or processes to one, unique label pointing to common features; once this is achieved, classificatory theories try to preserve complexity and to capture biological diversity by using a vast number of terms to identify phenomena. These classifications do not aim to reduce the number of labels used — this might happen as a result of the attempt to develop efficient tools for experimental research or, as we saw above, generalisations over species, but it is not a primary concern nor a necessary characteristic for these systems. The Gene Ontology for instance keeps diversifying and adding terms, rather than trying to reduce the terms it uses, so as to capture as precisely as possible existing knowledge about the specific processes or entities being investigated.

\section{(3) Explaining}

If we define explanations as descriptions employed to answer 'how' questions, following Michael Scriven (1969), then classificatory theories can definitely be seen as explanatory. For instance, the question 'how does gametogenesis work?' can be answered by quoting the definition of gametogenesis developed by the Gene Ontology; and one way to answer the 
question 'how does the chicken embryo develop bone structure' is to list the developmental stages through which bone structure is developed. By contrast, classificatory theories can hardly be seen as explanatory if, by explanation, we require reference to general explanatory principles or to highly general, law-like statements, as required for instance by the deductive-nomological model of explanation. Classificatory theories do not involve law-like, axiomatic statements such as the mathematical equations used in population genetics (e.g. Hardy-Weinberg's law of population equilibrium); they also differ from theories such as evolutionary theory, where a few basic principles provide the tenets for explaining the complex mechanisms of heredity. Strikingly, classificatory theories may display explanatory power, but do not set out to find explanations in the first place. Explanatory power is thus a secondary epistemic virtue in this kind of theorizing. . Other epistemic virtues take precedence in shaping this type of formalization: for instance, empirical accuracy, wide intelligibility, and heuristic value for future research.

\section{(4) Providing a grand vision guiding research}

Another characteristic often attributed to scientific theories is their role as providing grand ideas / general frameworks that can inspire and direct empirical investigations - as in the case of the central dogma in 1960s genetics, for instance. ${ }^{6}$ Again, classificatory theories do provide such a vision, but not in the ways that other types of theory do. Classificatory theories might commit to a specific vision of life, yet such a commitment is not central nor is it necessary to their development. Developing these theories might involve committing to other types of theories that provide all-embracing interpretations of reality, such as for instance evolutionary theory or, in the case of bio-ontologies, the principle of genetic conservation across species. However, such a

\footnotetext{
${ }^{6}$ For a discussion of the role of grand theories in data-intensive science, see Callebaut (2012).
} 
commitment is not necessary to their development, and classificatory theories rarely contribute to advancing such broad visions of the contents of biological knowledge. What classificatory theories do is embodying first and foremost a vision of how biological research should be conducted, rather than a vision of the content of biological knowledge itself. It is a heuristic role grounded primarily in methodological commitments, which may carry great epistemic and possibly ontological import depending on how (uncritically or critically) they are embraced by working scientists. Classificatory theory can express knowledge underlying specific research programs, thus helping to understand their assumptions and reflect on their implications. This does not require a grand vision for what biology should achieve and tell us about the world we live in. Rather, it provides a situated, localized expression of what (some) biologists believe about the world and how we go about studying it.

\section{Conclusion: What Is at Stake?}

A crucial objection that may be thrown against my arguments has to do with qualifying the results of (some) classificatory practices as a form of theory. What is at stake in the claim that classificatory practices constitute theorizing? Are classification systems not simply a component of scientific practice which affects, and yet does not constitute, theoretical knowledge? This objection seems especially strong in light of recent literature on the role played by elements other than theory, such as models, experiments and instruments, in shaping scientific research. Why are classifications of the type I described not a hitherto understudied type of model, or even background knowledge, rather than a form of theory? Would it not be enough to point to the 
important role played by classificatory activities in providing conceptual scaffolding for biological knowledge (as implied, for instance, by Wimsatt and Griesemer 2007)?

I believe that there are important reasons for pointing to classificatory activities as theorymaking, rather than simply as part of the background knowledge or modeling strategies used when theorizing. Probably the most important one is the role that such theory plays in delimiting the content and development of knowledge (what counts as biological insight, which form it can take); and as a target for critique and reference points for the construction of alternative accounts. Some classificatory systems systematically and synthetically express, rather than simply affect, knowledge obtained through scientific research, and they do it in a way that (1) is unique, since such knowledge is not formalized anywhere else in the same way; (2) has huge influence on knowledge-making practices; (3) enables experimenters to make sense of the results they obtain. At the same time, the conceptual framework that these theories provide only makes sense in light of specific traditions in handling data. The role played by what I call classificatory theories parallels Krakenau et al's discussion of what they call "bottom-theory" emerging from data handling practices: "Theory provides the basis for the general synthesis of models, and a means of supporting model comparisons and ideally establishing model equivalence" (Krakenau et al 2011: 272). Of course, classificatory theories are best understood in relation to the collection of models, instruments and commitments made by the researchers who produced it, as in the case of any scientific theory. However, they cannot be reduced to any of those other elements; and further, they provide a way to link and evaluate the epistemic results of using all those methods and tools to research nature. Articulating knowledge that enables scientists to assess and value their results is an achievement that goes well beyond listing a set of commonly used assumptions 
as a basis for further inquiry. In the latter case, existing knowledge is applied to put a given set of items into some order; in the former, existing knowledge is transformed and developed so as to facilitate the conceptual analysis of data. This is why the results of some classification systems should be viewed as theories rather than mere background knowledge - even if, as I have shown, this notion of theory differs from traditional depictions as a series of axioms or principles with great explanatory power and universal scope.

This brings me, in closing, to briefly reflect on an underlying commitment I made throughout this paper: the commitment to a pluralistic account of scientific theorizing. I do not believe that philosophical debates on the notion of theory should be tied to a uniform definition of what theory consists of in the whole of science, and I thus do not see the recognition of variability among types of theories, each structured and used in order to serve specific research goals, as problematic. On the contrary, the identification of different types of theories seems to me to open the way for an increasingly sophisticated philosophy of science, which is at once responsive to scientific practices and attentive to their epistemic and ontological context and implications. This argument runs parallel to the more popular argument about the importance of pluralism in models (e.g. Wimsatt 2007, Morgan and Morrison 1999, Leonelli 2007), styles of reasoning (Hacking 2002, Winther 2012) and ways of knowing (Pickstone 2000). In fact, the recent insistence on the plurality and scope of different forms of modeling has threatened to discard the notion of theory altogether as a relevant component of scientific practice, particularly in the case of philosophers sympathetic to the semantic view of theories as families of models (Giere 1999) or models as autonomous agents (Suarez and Cartwright 2008). In contrast to these accounts, I am convinced that the notion of theory has still an important role to play in scientific 
epistemology, and yet that our understanding of what counts as theory should shift to reflect the recent turn to scientific practice in analyzing other crucial components of science, such as models.

\section{Acknowledgments}

This research was funded by the ESRC as part of the ESRC Centre for Genomics in Society. I warmly thank the following individuals for very helpful discussions: the editors of this special issue Kim Sterelny and Massimo Pigliucci; the participants to the KLI workshop on 'The Meaning of Theory' (particularly Werner Callebaut and James Griesemer) and to the Biological Interest Groups at the University of Minnesota (particularly Alan Love and Bill Wimsatt) and the University of Exeter (particularly John Dupré, Staffan Müller-Wille and Berris Charnley); and Maureen O’Malley, Jane Lomax, Midori Harris and James McAllister.

\section{Bibliography}

Ashburner M, et al. (2000) Gene ontology: tool for the unification of biology. Nature Reviews Genetics 25(1): 25-29.

Bard JL, Rhee SY (2004) Ontologies in biology: design, applications and future challenges. Nature Review Genetics 5: 213-22.

Bogen J, Woodward J (1988) Saving the phenomena. Philosophical Review 97(3): 303-352. 
Bowker GC, Star SL (1999) Sorting Things Out: Classification and Its Consequences. Cambridge, MA: The MIT Press.

Brigandt I (2009) Natural kinds in evolution and systematics: metaphysical and epistemological considerations. Acta Biotheoretica 57: 77-97.

Callebaut W (2012) Scientific perspectivism: a philosopher of science's response to the challenge of big data biology. Studies in History and Philosophy of Science Part C: Studies in History and Philosophy of Biological and Biomedical Sciences 43 (1):69-80.

Crombie AC (1994) Styles of Scientific Thinking in the European Tradition: The History of Argument and Explanation Especially in the Mathematical and Biomedical Sciences and Arts. Gerald Duckworth \& Co Ltd.

Daston L (2004) Type specimens and scientific memory. Critical Inquiry, 31: 153-182.

Dewey J (1938) Logic: The Theory of Inquiry. New York: Holt Rinehart \& Winston.

Dupré J (1993) The Disorder of Things: Metaphysical Foundations of the Disunity of Science. London: Harvard University Press.

Dupré J (2001) In defense of classification. Studies in the History and Philosophy of the Biological and Biomedical Sciences, 32: 203-219.

Dupré J, O’Malley MA (2007) Metagenomics and biological ontology. Studies in the History and Philosophy of the Biological and Biomedical Sciences, 38: 834-846.

Giere RN (1999) Science Without Laws. Chicago, Chicago University Press. 
Griesemer JR (1996) Periodization and models in historical biology. In: Memoirs of the California Academy of Sciences, no. 20, New Perspectives on the History of Life (Ghiselin MT, Pinna G eds), 19-30. San Francisco: California Academy of Sciences.

Griesemer JR (2000) Development, culture and the units of inheritance. Philosophy of Science 67 [Supplement: Proceedings of the 1998 Biennial Meetings of the Philosophy of Science Association]: $348-368$.

Griesemer JR (2006) Theoretical integration, cooperation, and theories as tracking devices. Biological Theory, 1:4-7.

Hacking I (1992) The self-vindication of the laboratory sciences. In: Science as Practice and Culture (Pickering A ed), 29-64. Chicago: University of Chicago Press.

Hacking I (2002) Historical Ontology. Cambridge, MA: Harvard University Press.

Klein U, Lefèvre W (2007) Materials in eighteenth-century science: A historical ontology. Cambridge, MA: MIT Press.

Krakenau DC et al. (2011) The challenges and scope of theoretical biology. Journal of Theoretical Biology 276: 269-276.

Leonelli S (2007) What is in a model? Using theoretical and material models to develop intelligible theories. In: Modeling Biology. Structures, Behaviour, Evolution (Laubichler M, Muller GB eds). Vienna Series: MIT Press.

Leonelli S (2009) On the locality of data and claims about phenomena. Philosophy of Science 76(5): $737-749$. 
Leonelli S (2010) Documenting the emergence of bio-ontologies: or, why researching bioinformatics requires HPSSB, History and Philosophy of the Life Sciences 32(1): $105-126$.

Leonelli S (2012a) Classificatory theory in data-intensive science: the case of Open Biomedical Ontologies. International Studies in the Philosophy of Science.

Leonelli S (2012b) When Humans Are the Exception: Cross-Species Databases at the Interface of Clinical and Biological Research. Social Studies of Science 42(2):214-236.

Leonelli S, Diehl AD, Christie KR, Harris MA, Lomax J (2011) How the gene ontology evolves. BMC Bioinformatics 12(325): 1-7.

Love A (2009) Typology reconfigured: from the metaphysics of essentialism to the epistemology of representation. Acta Biotheoretica 57: 51-75.

McAllister JW (1997). Phenomena and patterns in data sets. Erkenntnis, 47: 217-228.

Minelli A (2003) The Development of Animal Form: Ontogeny, Morphology, and Evolution. Cambridge: Cambridge University Press.

Morgan M, Morrison M (1999) Models as Mediators. Cambridge: Cambridge University Press.

Morrison M (2007) Unifying Scientific Theories: Physical Concepts and Mathematical Structures. Cambridge: Cambridge University Press.

Müller-Wille S (2007) Collection and collation: theory and practice of Linnaean botany. Studies in the History and Philosophy of the Biological and Biomedical Sciences 38: 541-562. 
Müller-Wille S, Charmantier I (2012) Natural history and information overload: the case of Linnaeus. Studies in the History and Philosophy of the Biological and Biomedical Sciences.

Parker W (2009) Does matter really matter? Computer simulations, experiments and materiality. Synthese 169: 483-496.

Pickstone J (2000) Ways of Knowing: A New History of Science, Technology and Medicine. Chicago: Chicago University Press.

Richardson MK, Minelli A, Coates M, Hanken J (1998) Phylotypic stage theory. Trends in Ecology and Evolution 13: 158.

Rheinberger H-J (2011) Infra-experimentality: from traces to data, from data to patterning facts. History of Science 49: 337-348.

Reydon TAC (2010) Natural kind theory as a tool for philosophy of science. In: EPSA Epistemology and Methodology of Science: Launch of the European Philosophy of Science Association (Suárez M, Dorato M, Rédei M eds), 245-255. Dordrecht: Springer.

Scriven M (1969) Explanation in the biological sciences. Journal of the History of Biology 2(1): 187-198.

Smiths B, Ceusters W (2010) Ontological realism: a methodology for coordinated evolution of scientific ontologies. Applied Ontology 5:139-188.

Strasser BJ (2011) The experimenter's museum: GenBank, natural history, and the moral economies of biomedicine, 1979-1982. Isis 102: 60-96. 
Strasser BJ, de Chadarevian S (2011) The comparative and the exemplary: revisiting the early history of molecular biology. History of Science 49: 317-336.

Suarez M, Cartwright N (2008) Theories: Tools vs. models. Studies in the History and Philosophy of Modern Physics, 39: 62-81

Winther RG (2012) Interweaving categories: styles, paradigms, and models. Studies in History and Philosophy of Science, Part A.

Wimsatt WC (2007) Re-Engineering Philosophy for Limited Beings. Cambridge, MA: Harvard University Press.

Wimsatt WC and Griesemer JR (2007) Reproducing entrenchments to scaffold culture: The central role of development in cultural evolution. In: Integrating Evolution and Development: From Theory to Practice (Samsom R, Brandon R eds.) Cambridge: MIT Press, 227-323. 